Louisiana State University

LSU Digital Commons

Faculty Publications

Department of Biological Sciences

$1-1-2013$

\title{
Parental psychological control dimensions: Connections with Russian preschoolers' physical and relational aggression
}

\author{
David A. Nelson \\ Brigham Young University \\ Chongming Yang \\ Brigham Young University \\ Sarah M. Coyne \\ Brigham Young University \\ Joseph A. Olsen \\ Brigham Young University \\ Craig H. Hart \\ Brigham Young University
}

Follow this and additional works at: https://digitalcommons.Isu.edu/biosci_pubs

\section{Recommended Citation}

Nelson, D., Yang, C., Coyne, S., Olsen, J., \& Hart, C. (2013). Parental psychological control dimensions: Connections with Russian preschoolers' physical and relational aggression. Journal of Applied Developmental Psychology, 34 (1), 1-8. https://doi.org/10.1016/j.appdev.2012.07.003

This Article is brought to you for free and open access by the Department of Biological Sciences at LSU Digital Commons. It has been accepted for inclusion in Faculty Publications by an authorized administrator of LSU Digital Commons. For more information, please contact ir@lsu.edu. 


\title{
Parental psychological control dimensions: Connections with Russian preschoolers' physical and relational aggression ${ }^{\hbar}$
}

\author{
David A. Nelson *, Chongming Yang, Sarah M. Coyne, Joseph A. Olsen, Craig H. Hart \\ School of Family Life, Brigham Young University, United States
}

\section{A R T I C L E I N F O}

\section{Article history:}

Received 4 July 2011

Received in revised form 27 June 2012

Accepted 18 July 2012

Available online 17 October 2012

\section{Keywords:}

Psychological control

Relational aggression

Physical aggression

Russia

\begin{abstract}
A B S T R A C T
Parental psychological control generally consists of overinvolved/protective and critical/rejecting elements, both being linked to children's psychosocial maladjustment. The critical/rejecting element is multidimensional in nature, and few studies have explored this conceptual fullness. It is possible that some dimensions, if they can be statistically differentiated, are uniquely tied to various child behaviors. This may help resolve some of the inconsistency apparent across studies, such as studies of relational aggression. Accordingly, we examined the association between parental psychological control and childhood physical and relational aggression using a dimensional approach. Participants were 204 Russian preschoolers and their parents. The results revealed that dimensions of psychosocial control (i.e., shaming/disappointment, constraining verbal expressions, invalidating feelings, love withdrawal, and guilt induction) could be statistically differentiated, even though most dimensions tended to be significantly correlated. Furthermore, all dimensions, except for invalidating feelings, were significantly associated with childhood aggression, but predominantly in same-gender parent-child dyads.
\end{abstract}

(c) 2012 Elsevier Inc. All rights reserved.
Psychological control is the practice of parents who "manage their children's behavior through manipulation of their children's emotions, intrusion on children's autonomous activity, or restriction of their children's range of experiences" (McShane \& Hastings, 2009, p. 481). From toddlerhood through adolescence, studies show that psychological control is consistently associated with child maladjustment. In this paper, we focus on a preschool sample, a valuable age period in which to assess parenting correlates of child behavior. Specifically, children of this age may be more open to parental influence than children in later developmental periods.

A fair number of studies have assessed parental psychological control in the context of young children's internalizing and externalizing behaviors. In regard to internalizing problems, Rapee's (1997) review pointed to parental rejection and inappropriate control as key factors associated with children's anxiety and depression. In the past decade, studies of psychological control in early childhood have further confirmed Rapee's summary, with both overinvolved/protective and criti$\mathrm{cal} /$ rejecting elements of psychological control being consistently tied to anxiety and depression. For example, Bayer, Sanson, and Hemphill (2006) found that overinvolved/protective psychological control was

\footnotetext{
This research was supported by grants from the Kennedy Center for International Studies, the College of Family, Home, and Social Sciences, the Camilla Eyring Kimball Endowment, and the Family Studies Center at Brigham Young University. We also ac knowledge the support of a Mary Lou Fulton Young Scholar Award to the first author, and a Zina Young Williams Card Professorship for Craig H. Hart.

* Corresponding author at: School of Family Life, Brigham Young University, 2102C JFSB, Provo, UT 84602-0720, United States.

E-mail address: david_nelson@byu.edu (D.A. Nelson).
}

positively associated with the development of internalizing difficulties in two-year-olds. McShane and Hastings (2009) found maternal overprotection and paternal critical control to be associated with preschoolers' internalizing problems and anxiety. Moreover, children's anxious behaviors predicted parents' overprotection and critical control, suggesting a bidirectional influence at work. Beyond bidirectional influences, Mills et al. (2007) showed how the psychological status of the parent increases the chances of engagement in psychological control. In particular, proneness of parents to self-directed shaming may either produce parental anxiety or anger that is projected on to the child in either overprotective or critical/rejecting forms of psychological control.

Whereas studies of internalizing behaviors have considered both overprotective and critical/rejecting forms of psychological control, studies of children's externalizing behaviors have frequently focused on the latter. In particular, critical/rejecting psychological control may be considered a form of aggressive parenting that may encourage more of the same in children. This line of thinking is consistent with social learning theory, in which parents may serve as models of social behavior for their children. This seems particularly relevant when considering the prediction of different types of child aggression.

Specifically, in recent years, physical aggression has been differentiated from relational aggression (Crick \& Grotpeter, 1995) and similar constructs like indirect aggression or social aggression (Cairns, Cairns, Neckerman, Ferguson, \& Gariepy, 1989; Feshbach, 1969; Galen \& Underwood, 1997; Lagerspetz, Björkqvist, \& Peltonen, 1988). Relational aggression entails behaviors that seek to manipulate or impair social relationships. Common strategies include 
rumors, gossip, and social exclusion. Based on social learning theory, some researchers initially presumed that children reared by psychologically controlling parents may employ similar manipulative strategies with peers (Nelson \& Crick, 2002). Indeed, the nature of relational aggression appears to parallel some dimensions of psychological control. For example, such parents may threaten to withdraw love or attention unless a child is compliant with parental wishes. Such behavior mirrors the relationally aggressive child's threats to end a friendship unless a friend proves compliant. In contrast, spanking might be most predictive of physical aggression, as spanking models the resolution of problems via physical force. Accordingly, specific connections between forms of aversive control and aggression subtypes are expected.

A number of studies confirm that parental psychological control is related to children's relational aggression, even in non-Western cultures (e.g., Casas et al., 2006; Kuppens, Grietens, Onghena, \& Michiels, 2009; Loukas, Paulos, \& Robinson, 2005; Nelson \& Crick, 2002; Nelson, Hart, Yang, Olsen, \& Jin, 2006). However, both physical and relational aggression are usually associated with both spanking and psychological control, so the theoretical specificity (suggested above) is lacking (cf., Kuppens et al., 2009). The gist is that aversive parenting models aggressive behavior more generally.

Consistent with prior work, the present study is predominantly framed by this social learning perspective. However, other theoretical perspectives have been proposed to explain the association between parental psychological control and children's relational aggression (Kuppens et al., 2009; Soenens, Vansteenkiste, Goossens, Duriez, \& Niemiec, 2008). In particular, a parent's use of coercive or psychological control may weaken the attachment relationship between parent and child, thereby denying the child of a sense of acceptance in this formative relationship. Relational insecurity may generalize to anxiety and accordingly precipitate difficulties in peer relationships. This perspective is consistent with attachment theory (Bowlby, 1988) and self-determination theory (Deci \& Ryan, 2000), both of which propose a fundamental human need for relatedness-close, emotionally secure relationships. The child may inappropriately cope with relationship insecurity by lashing out at peers (e.g., preemptively deterring aggression and threats to their social status; Soenens et al., 2008).

Alternatively, social-information processing theory (Crick \& Dodge, 1994) suggests that children's aggressive behavior is often the result of the unique manner in which children process social information as they interact with peers. For example, children may exhibit a hostile attribution bias, in which they perceive provocation when the true intent of the supposed provocateur is ambiguous (and may be benign; Nelson, Mitchell, \& Yang, 2008). Coercive and controlling parenting may promote these social cognition difficulties, and the child's subsequent aggressive behavior toward peers (Nelson \& Coyne, 2009; Weiss, Dodge, Bates, \& Pettit, 1992).

\section{Dimensionality of critical/rejecting psychological control}

When Barber (1996) renewed the study of parental psychological control, he conceptualized it as multidimensional. In particular, he described the critical/rejecting side of psychological control with six different dimensions. Love withdrawal and erratic emotional behavior, for example, both reflect dimensions in which feelings of parental acceptance are manipulated. Other dimensions include invalidation of the child's feelings (e.g., telling the child how to feel or think) or constraining their verbal expressions (e.g., speaking for the child). These actions communicate to the child that their feelings and verbal expressions are not valued. The child's sense of self may accordingly be undermined. Parents may also induce hurt feelings or shame in the child through the use of negative criticism (shame, disappointment, personal attack) and guilt induction. For example, parents may continually remind the child of all the sacrifices they have made for the child, hoping thereby to coerce a child's obedience to the parent's wishes. Together, these dimensions compose a substantial portion of psychological control construct.

Despite the initial emphasis on multidimensional nature of psychological control, few studies have adequately approached measurement of the construct in this manner. Barber's (1996) 16-item psychological control scale (youth self-report) is only composed of two to three items per each of the dimensions described above. This limited number of items may hamper effective factorial assessment of whether dimensions may be considered independently. Indeed, Barber's factor analysis of the 16-item measure reduced the final set to 8 items, which represented the constraining verbal expressions, invalidating feelings, and love withdrawal dimensions. Accordingly, use of the 8-item measure does not reflect half of the originally proposed dimensions of psychological control. Other studies of psychological control have followed suit, with psychological control scales that reflect a selected crosssection of the dimensions, rather than the full range of dimensions (e.g., Hart, Nelson, Robinson, Olsen, \& McNeilly-Choque, 1998; Nelson \& Crick, 2002).

Accordingly, it may be argued that psychological control has not been explored in its conceptual fullness. With the narrow scope of items in most studies, it is understandable that psychological control is often empirically reduced to a unidimensional structure (although an adequate Cronbach's alpha is hardly sufficient evidence of the unidimensional nature of a construct). Alternatively, a larger bank of psychological control items might allow for a dimensional assessment of psychological control. Accordingly, the present study departs from past practice by assessing psychological control with sufficient items for confirmatory factor analysis and the ability to reduce an item bank down to dimensions that are expected to be significantly correlated yet statistically distinguished. We then consider whether greater predictive precision might be obtained with such an approach. Specifically, the narrow measurement of psychological control in prior studies may have hampered our knowledge of its actual associations with child physical and relational aggression.

Although the majority of existing studies have established a link between parental psychological control and relational aggression, the first, conducted with a Russian preschool sample (Hart et al., 1998), is an exception. The focus on psychological control in that study was in keeping with Bronfenbrenner's (1970) earlier observations of Soviet childrearing, wherein he described the presence of "love-oriented" discipline. Results of the study showed that psychological control could indeed be successfully measured in a contemporary Russian sample of preschool parents. Contrary to expectations, however, parental psychological control was predictive of physical but not relational aggression. Hart et al. used a composite psychological control scale which included items representing love withdrawal, guilt induction, and negative criticism (shaming/disappointment). Accordingly, the potentially unique influence of the three individual dimensions was diluted by mixing them, and other dimensions of psychological control (i.e., erratic emotional behavior, constraining verbal expressions, invalidating feelings), which may be significantly associated with relational aggression, were absent from the scale. Thus, it remains to be seen whether specific dimensions of parental psychological control might be more useful in prediction over an omnibus scale (particularly one of limited breadth). Accordingly, in this study, we revisit the Russian preschool data with a dimensional approach. The emphasis of this paper, then, is not so much on a cultural context (in this case, Russia) as it is on the predictive utility of a dimensional approach to psychological control.

Our approach builds on one prior study with similar goals. Working with a group of U.S. preschoolers and their parents, Casas et al. (2006) formed five dimensions of psychological control (erratic emotional behavior, love withdrawal, guilt induction, invalidating feelings, and directiveness). Two other dimensions (i.e., personal attack, constraining verbal expressions) were also assessed but did 
not have satisfactory scale reliabilities and were therefore excluded. Results of multiple regression analyses found few dimensions to be significantly predictive of child aggression, with most associations obtained for girls.

However, the present study brings a more sophisticated dataanalytic approach to the problem. Casas et al. (2006) did not report the correlations between the different dimensions, although as components of the same overarching parenting style we may expect moderate to high correlations (Barber, 1996). Accordingly, beyond reports of coefficient alpha for the respective scales (Casas et al., 2006), statistical distinctiveness of dimensions of psychological control has yet to be directly tested. The present study is therefore designed to assess the statistical distinctiveness and predictive significance of psychological control dimensions, using a latent variable structural equation modeling (SEM) approach. Given the ability of an SEM approach to account for measurement error, it may also yield more reliable estimates of the associations of interest. If various dimensions of psychological control can be statistically differentiated, we will then assess the associations of the psychological control dimensions with subtypes of childhood aggression (i.e., physical and relational) via their latent correlations.

We also consider both parent and child gender in the association between parental psychological control and child aggression. Consistent with social learning theory, prior research often suggests that children prefer to imitate adults of the same gender, who are more similar than dissimilar to them (e.g., Chang, Schwartz, Dodge, \& McBride-Chang, 2003; Deater-Deckard \& Dodge, 1997). Indeed, the dimensional approach of Casas et al. (2006) predominantly yielded associations for same-gender dyads. Other studies have yielded mixed results, however (e.g., Hart et al., 1998; Kuppens et al., 2009; Nelson \& Crick, 2002; Nelson et al., 2006), so we further explore these associations with our dimensional approach.

\section{Hypotheses}

Our first hypothesis was that we expected the psychological control dimensions to be moderately to highly correlated yet statistically differentiated. This is not unlike the clustering of categories of aversive parenting (e.g., spanking and psychological control), which are nonetheless considered separately in research (e.g., Nelson \& Crick, 2002; Nelson et al., 2006). We statistically compare multidimensional and unidimensional models of psychological control in this regard.

Second, based on social learning theory, we expected certain dimensions to more likely model aggressive interaction patterns. We were particularly focused on relational aggression, given the minimal research dedicated to familial correlates (relative to physical aggression). Accordingly, we expected dimensions of psychological control which clearly manipulate the parent-child relationship to be particularly associated with relational aggression, given the behavioral similarity. Love withdrawal, in particular, is similar to the practice of relationally aggressive individuals. The conditional nature of the parent-child relationship suggested by dimensions like erratic emotional behavior or negative criticism would also appear to mirror relational aggression. Thus, we expected more associations between these psychological control dimensions and relational aggression than physical aggression.

\section{Method}

Setting

The participants in this study were parents and their preschool children living in Voronezh, Russia, a city of approximately 900,000 people located 280 miles south of Moscow. This provincial city was closed to foreigners during the Soviet period and, accordingly, the entire sample of parents and their children were ethnic Russians.
Although this $100 \%$ Russian sample is representative of the Voronezh region, it is not typical of the more cosmopolitan populations of the largest cities such as Moscow and St. Petersburg, where minority populations are evident. Voronezh is, however, reflective of typical Russian life beyond the Western-influenced atmospheres of Moscow and St. Petersburg.

\section{Participants}

The sample was composed of 207 Russian preschoolers and their parents (207 mothers, 167 fathers). Thirty-two of these families were led by single mothers, and fathers refused to participate in eight families. These families were recruited from a potential sample of 255 eligible families (effective consent rate of 81.2\%), and were drawn from 15 classrooms in three separate nursery schools located in close proximity to one another in a central section of the city. In three families, neither the father nor the mother completed the psychological control measure, and these families were removed from further analysis (effective sample size of 204 mothers and 164 fathers). The sample was composed of 96 boys and 108 girls, with a mean age of 5.1 years $(S D=0.72)$. The parents of these children were generally well educated. Mothers, on average, had 14 years 11 months $(S D=2.34)$ of education and fathers averaged 14 years 6 months $(S D=2.42)$ of education. The participating preschooler was the only child in $69 \%$ of families, with an additional 30\% having two children (only $1 \%$ with more than two). Given that we had teacher reports of aggression for 204 children, missing parental data was accounted for in the analyses below using full information maximum likelihood estimation.

\section{Procedure}

The study was approved in advance by the university IRB of the authors. The research proposal was then translated and presented to the lead administrators of the Voronezh school district. After a review, district administrators gave permission for the study to proceed. We were allowed to approach the three nursery schools and ask the school directors and teachers for their willingness to facilitate the research project. All nursery school personnel were enthusiastic to assist. Russian schools act in loco parentis, meaning that we were not allowed to obtain written evidence of parental permission for the study. However, information meetings were conducted with parents at the beginning of the study in which the details of the study were explained and parents were assured of confidentiality. They were also told that they could withdraw themselves or their child from the study at any time. Children were also allowed to withdraw from any classroom assessment if they chose to do so.

Nearly all mothers and fathers who attended the information meeting (not all parents attended) were inclined to participate in the study. Accordingly, we then gave instruction regarding questionnaires they would complete over the course of the study (each questionnaire was reviewed, one by one, and the psychological control measure was just one of many measures described and administered). Accordingly, many other measures of marital, parent-child, and family variables are not included in this particular study. The parents were told that two additional weekly meetings would be held, and with each meeting they would receive a questionnaire packet to complete at home (for a total of three questionnaire packets completed over a three-week period). Parents returned the first completed packet at the second meeting and were instructed in regard to the second packet questionnaires, and so forth. Parents were given separate mother and father packets and instructed to complete measures independent of their spouse. Parents were instructed that there were no right or wrong answers, and they should individually answer each question according to their own personal feelings about self, spouse or child. During this time, preschool teachers supplied the ratings of the child's aggressive behavior with peers at school (which are used in this study). Data were collected at 
the very end of the school year, in the spring, to ensure that teachers were thoroughly familiar with each child they rated.

\section{Measures}

\section{Teacher ratings of aggressive behavior}

Teacher ratings were employed to obtain a measure of physical and relational aggression for each child (see Table 1 for associated items). These measures were derived from preschool measures previously employed with North American samples, with the measures reflecting satisfactory reliability and validity (Crick, Casas, \& Mosher, 1997; McNeilly-Choque, Hart, Robinson, Nelson, \& Olsen, 1996). The items were successfully forward- and back-translated by Russian linguists. Study authors consulted with translators in regard to difficult-to-translate items. Russian teachers rated the frequency of specific aggressive behaviors using a 3-point scale (never, sometimes, often). Teacher ratings of aggression are generally viewed as a reliable assessment tool (Archer \& Coyne, 2005) and tend to be moderately correlated with other forms of measurement (i.e., peer ratings; Nelson, Robinson, \& Hart, 2005).

\section{Parental self-reports of dimensions of psychological control}

Parents completed a self-report measure of critical/rejecting forms of psychological control, with items representing multiple dimensions of psychological control. Similar to the teacher measures, the psychological control items were successfully forward- and back-translated by Russian linguists. The items were derived from the Parental Psychological Control measure (PPC; Hart \& Robinson, 1995), which is composed of 37 items (adapted from existing instruments or of new creation). These items cover the following psychological control dimensions: constraining verbal expressions (4 items), invalidating feelings (3 items), erratic emotional behavior (6 items), love withdrawal (4 items), guilt induction ( 8 items), and negative criticism (shame, disappointment, personal attack; 11 items). Mothers and fathers rated their own psychologically controlling behavior with their preschool child on a 5-point scale ranging from 1 (never) to 5 (always). This measure has been used successfully in assembling omnibus psychological control scales in previous research (e.g., Hart et al., 1998, Nelson \& Coyne, 2009; Nelson \& Crick, 2002) but it has never been subjected to multidimensional factor analysis like what we conducted here. We envisioned that a substantial item bank would yield a sufficient number of items for each dimension following factor analysis.

Table 1

Standardized factor loadings of the aggression constructs.

\begin{tabular}{|c|c|c|}
\hline \multirow[b]{2}{*}{ Constructs and contents } & \multicolumn{2}{|c|}{ Loadings } \\
\hline & Boys & Girls \\
\hline \multicolumn{3}{|l|}{ Physical aggression } \\
\hline Hits, kicks, or pushes to get something he/she wants. & .90 & .87 \\
\hline $\begin{array}{l}\text { Threatens to push a peer off a toy (e.g., tricycle, play house) or ruin } \\
\text { what peer is working on unless he/she shares. }\end{array}$ & .95 & .93 \\
\hline $\begin{array}{l}\text { Pushes or hits peers to get even for something that was done } \\
\text { accidentally. }\end{array}$ & .85 & .85 \\
\hline $\begin{array}{l}\text { Uses hostile means to keep other children from having what he/she } \\
\text { has (e.g., swing). }\end{array}$ & .93 & .93 \\
\hline $\begin{array}{l}\text { Throws things at other children when he/she doesn't get his/her } \\
\text { own way. }\end{array}$ & .85 & .76 \\
\hline \multicolumn{3}{|l|}{ Relational aggression } \\
\hline Tells other children not to play with someone. & .80 & .84 \\
\hline $\begin{array}{l}\text { Tries to get others to dislike a peer (e.g., whispering mean things } \\
\text { about the child behind their back). }\end{array}$ & .90 & .93 \\
\hline Tries to exclude other children who want to play. & .88 & .86 \\
\hline $\begin{array}{l}\text { Tells other children that they can't play with the group } \\
\text { unless they do what the group wants them to do. }\end{array}$ & .94 & .97 \\
\hline $\begin{array}{l}\text { Tells a peer that they won't be invited to a birthday party unless } \\
\text { he/she does what the child wants. }\end{array}$ & .88 & .76 \\
\hline Says, "I won't be your friend" to peers "if you don't do things my way." & .96 & .91 \\
\hline
\end{tabular}

Analytical plan

We followed two steps in conducting our analyses, consistent with the order of our hypotheses enumerated earlier. First, two sets of confirmatory factor analysis (CFA) were conducted in order to examine the quality of measurement of the primary constructs (child aggression, psychological control dimensions). These measurement models were conducted using the Mplus statistical program (Muthén \& Muthén, 2001), with methods for analyzing categorical data. In these models, we also note that factorial invariance (equality of the corresponding factor loadings) was assessed across child gender (boys vs. girls) using multiple group analysis, and across parent gender (mothers vs. fathers) using dyadic analysis. In each case, this was accomplished by comparing the baseline unconstrained model to a competing model in which factor loadings are constrained to be equal across child gender groups or across the gender of parental dyad members. Factorial invariance is emphasized as it allows for more direct comparison of results across the different parent-child dyads (Horn \& McArdle, 1992; Widaman \& Reise, 1997). Moreover, the resulting measurement models also provided the basis for assessing the statistical distinctiveness of correlated latent variables (Nelson et al., 2006), particularly in the comparison of a multidimensional and a unidimensional model of psychological control. Second, with appropriate measurement of the latent constructs established, the child and parent constructs were then combined in an additional CFA in order to assess the latent correlations between parenting and child aggression.

All of these models were evaluated and compared using the model chi-Square value and appropriate chi-Square difference tests, along with other fit measures including the Comparative Fit Index (CFI), the Tucker-Lewis Index (TLI) and the Root Mean Square Error of Approximation (RMSEA). For both the CFI and TLI, values should exceed .90 in order to establish acceptable model fit, with values above .95 representing the more optimal range. In contrast, RMSEA values under .05 indicate excellent fit, and a value between .05 and .08 reflects a reasonable fit. Because we use weighted least squares estimation for ordinal data, chi-Square difference tests are conducted using techniques provided by the Mplus software.

In regard to reliability of measurement, we note that coefficient alpha is not typically used with latent variables in confirmatory factor analysis as latent variables are not summed multiple-item scales and such values cannot, therefore, be calculated. In addition, coefficient alpha is considered to be a questionable indicator of reliability (Komaroff, 1997; Schmitt, 1996), particularly for scales composed of few items (i.e., coefficient alpha is enhanced by a greater number of items in a scale). Instead, standardized factor loadings of .40 and above of the observed variables are considered evidence of sufficient reliability for latent constructs in SEM (for samples of 150 or more; Stevens, 1996). This is reflected in the tables below.

\section{Results}

\section{Measurement model of childhood aggression subtypes}

In regard to the measurement of childhood aggression, the multiplegroup baseline CFA measurement model of the aggression constructs, allowing different factor loadings for boys and girls, fit the data satisfactorily; $\chi^{2}(95)=135.95, p<.01, \mathrm{CFI}=.99, \mathrm{TLI}=.99$, RMSEA $=.07$. The constrained model, $\chi^{2}(104)=144.28, p<.01$, CFI $=.99$, TLI $=.99$, RMSEA $=.06$, showed no significant decrement in the goodness of fit; $\chi_{\text {diff }}^{2}(9)=10.30 p=.33$. Thus, invariant measurement of the aggression constructs was obtained for boys and girls. The standardized factor loadings of the baseline model are shown in Table 1 for both boys and girls.

Due to the high latent correlation of the two aggression constructs ( $\varphi=.81$ in boys and .85 in girls); an additional model using similar indicators to reflect a single aggression construct was compared 
with the baseline (two-construct) model (this comparison was conducted for boys and girls combined). The chi-square difference test showed that the single construct model, $\chi^{2}(108)=225.53, \mathrm{p}<$ .001 , CFI $=.98$, TLI $=.98$, RMSEA $=.10$ did not fit the data as well as the two-construct model; $\chi^{2}$ diff $(4)=38.18, p<.001$. Thus, the two constructs were statistically distinguished, though they are highly correlated (Nelson et al., 2006). Furthermore, for the sake of comparison with earlier work, we computed the equivalent Pearson correlations for the aggression scale scores ( $r s=.69$ and .72 for boys and girls, respectively). These are fairly similar to values reported in prior research regarding physical and relational aggression (McNeillyChoque et al., 1996; Nelson et al., 2005). Despite the relatively high correlation, numerous examples of criterion validity have distinguished relational and physical aggression across a significant number of studies (e.g., Nelson et al., 2006).

\section{Measurement model of parental psychological control}

We noted earlier that this measure of psychological control has never been subjected to factor analysis regarding multiple dimensions of psychological control (of the critical/rejecting type). Prior to the CFA, all 37 items were included in a basic exploratory factor analysis. In this analysis, a number of items had substantial cross-loadings on multiple dimensions or did not load on any particular dimension. Accordingly, such items were gradually pruned from the model. In this process, we also found that erratic emotional behavior would only converge as a two-item measure. Since two-indicator constructs are not ideal in CFA, we elected to drop erratic emotional behavior from the considered dimensions. Accordingly, when sufficient stability was evident in the initial EFA model, we composed the CFA and retained five of six original dimensions in the final model (21 total items with 3-5 items per dimension).

The resulting dyadic baseline measurement model (unconstrained model with different factor loadings for mothers and fathers) fit the data satisfactorily; $\chi^{2}(753)=855.51, p<.01$, CFI $=.95$, TLI $=.94$, and RMSEA $=.03$. In addition to the factor loadings and factor variances and covariances, this model also included correlations between the errors of the corresponding items for mothers and fathers. Furthermore, a model in which the factor loadings of the maternal and paternal psychological control dimensions were constrained to be equal, $\chi^{2}(769)=856.24, p<.05$, CFI $=95$, TLI $=.95$, RMSEA $=$ .02 , showed no significant decrement in model fit, $\chi_{\text {diff }}^{2}(16)=$ $10.51, p=.84$, implying that the factor loadings were invariant across mothers and fathers. The standardized factor loadings of the baseline model are listed in Table 2.

\section{Intercorrelations and statistical distinctiveness of parenting constructs}

The latent correlations between the various parenting constructs are listed in Table 3. Relatively high latent correlations were obtained within informant for several sets of psychological control dimensions. In particular, guilt induction was highly correlated with constraining verbal expressions for fathers and guilt induction was highly correlated with love withdrawal for mothers. Due to the relatively high latent correlations between a variety of psychological control dimensions, a unidimensional model was estimated and compared with the baseline (five-factor) model.

Thus, in the first model comparison, a model treating psychological control as unidimensional construct for both mothers and fathers was compared to the baseline model. In both of these models, the corresponding factor loadings for mothers and fathers were constrained to be equal. The chi-square difference showed that the baseline five-factor model (for both mothers and fathers) $\chi^{2}(817)=1001.24$, $\mathrm{CFI}=.90 \mathrm{TLI}=.90 \mathrm{RMSEA}=.03$ fit the data better; $\chi_{\text {diff }}^{2}(48)=$ $142.28, p<.001$. Accordingly, though significantly correlated, the

Table 2

Standardized factor loadings of the dimensions of psychological control.

\begin{tabular}{lcc}
\hline \multicolumn{1}{c}{ Constructs and content } & \multicolumn{2}{c}{ Loadings } \\
\cline { 2 - 3 } & Mothers & Fathers \\
\hline Shaming/disappointment & & \\
I try to change our child. & .45 & .55 \\
I tell our child that his/her behavior was dumb or stupid. & .51 & .55 \\
I act disappointed when our child misbehaves. & .63 & .70 \\
I tell our child that he/she should be ashamed when & .67 & .65 \\
he/she misbehaves. & & .61 \\
I let our child know how disappointed we are when & .69
\end{tabular}

he/she misbehaves.

Constraining verbal expression

I change the subject whenever our child has something to say.

I interrupt our child when he/she is speaking.

I finish our child's sentence whenever he/she talks.

I don't pay attention when our child is talking to us.

$\begin{array}{ll}.44 & .41 \\ .47 & .62 \\ .55 & .63 \\ .40 & .50\end{array}$

Invalidating feelings

I try to change how our child feels or thinks about things.

I want to control whatever our child does.

I would like to tell our child how to feel or think about things.

.54

.50

.57

.54

Love withdrawal

I avoid looking at our child when our child has disappointed me.

I ignore our child when he/she tries to get attention.

If our child has hurt our feelings, I stop talking to our child until our child pleases me again.

I am less friendly with our child if our child does not see things my way.

Guilt induction

$\begin{array}{llll}\text { I tell our child he/she is not as good as we were growing up. } & .51 & .73\end{array}$

I make our child aware of how much we sacrifice or do for him/ $\quad .52 \quad .69$

her.

I say, "If you really care for me, you would not do things that $\quad .67 \quad .66$ cause me to worry."

I make our child feel guilty when our child does not meet our $\quad .61 \quad .64$ expectations.

I bring up our child's past mistakes when criticizing him/her.

.55 .56

different parenting constructs were statistically distinguished for both mothers and fathers.

Latent correlations between parental psychological control and childhood aggression

Finally, the latent correlations of parental dimensions of psychological control with child aggression subtypes were examined within a final CFA containing all the constructs in the study. In prelude to a discussion of this CFA model, we note that skewness of a few parenting items became an issue when we separated data in order to look at

Table 3

Latent correlations among the parenting constructs.

\begin{tabular}{llllllllll}
\hline & FSD & FCVE & FIF & FLW & FGI & MSD & MCVE & MIF & MLW \\
\hline FCVE & $.54^{* * *}$ & & & & & & & & \\
FIF & $.73^{* * * *}$ & .24 & & & & & & & \\
FLW & $.68^{* * *}$ & $.73^{* * *}$ & $.36^{* *}$ & & & & & & \\
FGI & $.73^{* * *}$ & $.83^{* * *}$ & $.38^{* * *}$ & $.77^{* * *}$ & & & & & \\
MSD & $.38^{* * * *}$ & $.31^{* *}$ & $.27^{*}$ & $.38^{* * *}$ & $.25^{* *}$ & & & & \\
MCVE & .23 & $.39^{*}$ & .18 & $.38^{* *}$ & .00 & $.24^{*}$ & & & \\
MIF & $.31^{* *}$ & .18 & .15 & $.33^{*}$ & .18 & $.73^{* * *}$ & $.46^{* *}$ & & \\
MLW & $.32^{* *}$ & $.41^{* * *}$ & .13 & $.57^{* * *}$ & $.44^{* * *}$ & $.73^{* * *}$ & $.66^{* * *}$ & $.66^{* * *}$ & \\
MGI & $.46^{* * * *}$ & $.50^{* * *}$ & .18 & $.64^{* * *}$ & $.40^{* * *}$ & $.69^{* * *}$ & $.66^{* * *}$ & $.54^{* * *}$ & $.85^{* * * *}$
\end{tabular}

Notes: (F)SD = (Father's) Shaming/Disappointment; (M)CVE = (Mother's) Constraining Verbal Expressions; IF = Invalidating Feelings; $\mathrm{LW}=$ Love Withdrawal; $\mathrm{GI}=$ Guilt Induction.

${ }^{*} p<.05$.

** $p<.01$.

$p<.001$. 
latent correlations by gender of child (this problem was not evident in the earlier parenting CFA with parents of boys and girls merged together). For four items (all representing different dimensions), very few respondents used the highest category (5 on a 5-point scale) in their self-reports of parenting for either boys or girls. Accordingly, when the data was analyzed separately by gender of child, a situation arose in which a few parents used the highest category for a few children of one gender but not the other (e.g., parents of boys using categories 1-4 and parents of girls using $1-5$, even if the 5th category was used very sparingly for girls). Accordingly, the categorical analysis of these items could not proceed by gender of child without an appropriate adjustment. In the four cases where this happened, the 5th category responses were recoded to be category 4 , so that parallel category structures were available for parents of boys and girls (and analysis by gender of child could proceed).

After these minor adjustments, the CFA model fit the data adequately; $\chi^{2}(2643)=2804.93, p=.01$, CFI $=.96$, TLI $=.96$, RMSEA $=.03$. The latent correlations between (physical and relational) aggression and dimensions of psychological control were estimated for each parentchild dyad and are reported in Table 4. For father-son dyads, physical aggression was correlated with shaming/disappointment $(\varphi=.42)$, constraining verbal expressions $(\varphi=.30)$, love withdrawal $(\varphi=.49)$, and guilt induction $(\varphi=.38)$. Relational aggression was associated with paternal shaming/disappointment $(\varphi=.25)$, constraining verbal expressions $(\varphi=.37)$, love withdrawal $(\varphi=.38)$, and guilt induction $(\varphi=.27)$. For mother-son dyads, physical aggression was associated with shaming/ disappointment $(\varphi=.24)$, constraining verbal expressions ( $\varphi=.37$ ), and love withdrawal $(\varphi=.36)$, whereas relational aggression was associated with maternal shaming/disappointment $(\varphi=.28)$ and love withdrawal $(\varphi=.22)$. For father-daughter dyads, only one association emerged between physical aggression and guilt induction $(\varphi=.19)$. In mother-daughter dyads, physical aggression was associated with shaming/disappointment $(\varphi=.29)$, love withdrawal $(\varphi=.26)$, and guilt induction $(\varphi=.35)$. Relational aggression was correlated with maternal shaming/disappointment $(\varphi=.31)$ and guilt induction $(\varphi=.27)$.

\section{Discussion}

The findings of this study provide some unique insights into the nature of rejecting forms of psychological control and basic associations of the resulting dimensions with childhood aggression. As one of the few studies to attempt a dimensional approach in defining psychological control, this study predominantly serves as a springboard for future research. The CFA results show that dimensions of parental psychological control, despite relatively high correlations between some dimensions, can generally be statistically distinguished. Accordingly, it is possible to assess the associations of each dimension (with the exception of erratic emotional behavior, in our case) with child outcomes such as physical and relational aggression. Moreover, the instability of the erratic emotional behavior items may not be all that surprising, in that emotion swings may predominate and motivate the unconventional methods that broadly characterize rejecting forms of psychological control. However, further research will be needed to clarify this association with other samples, both within Russia and across other cultures. It is also notable that there was considerable diversity in the intercorrelations between individual psychological control dimensions for mothers and fathers. Specifically, some dimensions were highly correlated whereas others were uncorrelated. Accordingly, it appears that parents may favor some forms of psychological control over others, and perhaps no parent engages in all forms of psychological control (at least in the context of self-rated control).

As expected, psychological control dimensions were associated with teacher-rated aggression. Accordingly, these findings provide the first evidence that psychological control is associated with relational aggression in a Russian preschool sample (cf., Hart et al., 1998). A more thorough investigation of psychologically controlling behaviors clearly made a difference in the associations obtained, as four of the five dimensions were significantly associated with aggression in children. Latent correlations ranged from modest to moderate in size (ranging from .19 to .49). Invalidating feelings in parents was the only dimension not associated with either form of aggression in children. This broadly underscores the assertion of Barber $(1996,2002)$ that psychological control is uniformly considered to be negative parental control. This is especially considered important in the lives of adolescents, for which parental control issues interact with their quest for a sense of identity. The findings of this study, however, accentuate the fact that psychological control can be associated with negative child outcomes as early as preschool (Nelson et al., 2006). Accordingly, parental control issues in adolescence are likely the accumulation of more than a decade of negative parental control attempts.

The interest in potential parenting effects on children's peer behaviors is in alignment with the basic tenets of social learning theory (Bandura, 1971), where it is presumed that parents model aversive interactions which are then mimicked in the child's interactions with peers (Nelson et al., 2006, 2008). Accordingly, we originally hypothesized that shaming/disappointment, erratic emotional behavior, and love withdrawal would be dimensions of psychological control most likely to associate with relational aggression in Russian preschoolers. This emphasis is also consistent with initial studies of parenting and relational aggression which hypothesized some predictive specificity between forms of aversive parental control and child aggression subtypes. Parental corporal punishment was expected to uniquely predict child physical aggression, whereas parental psychological control was expected to uniquely predict child relational aggression. Nonetheless, this study adds further evidence that psychological control is more generally predictive of both forms of aggression (cf., Kuppens et al., 2009). Shaming/disappointment and love withdrawal were indeed key parental correlates for both boys and girls, but not with any real specificity regarding aggression subtypes. In fact, by a small margin, psychological control dimensions were more likely to be associated with physical rather than relational aggression overall ( $11 \mathrm{vs.} 8$ associations). Thus, the pattern of findings (for boys, in particular) for most dimensions appeared to be that of general association with both forms of aggression, rather than one or the other. In sum, aversive parenting is associated with child hostility, regardless of the type of parenting employed. Physical and relational aggression are also highly correlated, and it may therefore be a stretch to expect such specificity

Table 4

Estimated latent correlations $(\phi)$ between aggression subtypes and dimensions of psychological control, by gender of child.

\begin{tabular}{|c|c|c|c|c|c|c|c|c|c|c|c|}
\hline & & FSD & FCVE & FIF & FLW & FGI & MSD & MCVE & MIF & MLW & MGI \\
\hline \multirow[t]{2}{*}{ Boys } & Physical aggression & $.42^{* * *}$ & $.30^{*}$ & .13 & $.49^{* * *}$ & $.38^{* *}$ & $.24^{*}$ & $.37^{*}$ & .14 & $.36^{* *}$ & .05 \\
\hline & Relational aggression & $.25^{*}$ & $.37^{* *}$ & .05 & $.38^{* *}$ & $.27^{*}$ & $.28^{*}$ & .18 & .12 & $.22^{*}$ & .07 \\
\hline \multirow[t]{2}{*}{ Girls } & Physical aggression & .16 & .14 & -.18 & -.06 & .19 & $.29^{* *}$ & .09 & -.01 & $.26^{*}$ & $.35^{* *}$ \\
\hline & Relational aggression & .00 & .03 & -.08 & .14 & .15 & $.31^{* *}$ & .03 & .07 & .20 & $.27^{* *}$ \\
\hline
\end{tabular}


Guilt Induction.

$\begin{array}{rl}{ }^{*} & p<.05 . \\ { }^{* *} & p<.01 . \\ * * * & p<.001 .\end{array}$ 
in associations with parenting. The more interesting question seems to be how parenting has its influence across the different parent-child gender dyads.

In particular, psychological control appeared to be most prominently associated with childhood aggression in the context of same-gender parent-child relationships (accounting for two-thirds of the statistically significant findings). In this regard, the lack of father-daughter associations appears to contrast with previous studies that have identified such an association in both U.S. (Nelson \& Crick, 2002) and Chinese (Nelson et al., 2006) samples. However, it is important to keep in mind that these prior studies did not utilize a dimensional approach, so the comparison is somewhat limited. These findings suggest, however, that the effects of psychological control may differ across cultures as parents engage in different levels of such parenting or may find their greatest influence or interaction with same-gender or opposite-gender children. It remains to be seen how these patterns may correspond with cultural expectations regarding parenting and child gender. Additional study of parenting norms within each culture, and the basic nature of gendered parent-child relationships, should provide the insight that is needed. To date, very few studies have attempted to document normative patterns of parent-child interaction in Russian samples, so we can only speculate.

As noted earlier, social learning theory and previous research both suggest that same-gender parent-child relationships may be the most influential contexts for a child to learn or imitate behavior. For example, children are more likely to model physical aggression when it is demonstrated by the same-gender parent (e.g., Davis, Hops, Alpert, \& Sheeber, 1998). Although the majority of findings suggested the possibility of same-sex modeling, findings for boys suggested less specificity, with quite a few associations emerging in mother-son dyads. With the exception of guilt induction, there was also considerable parity in the associations between boys' aggression and the psychological control of mothers versus fathers.

Accordingly, social learning theory does not appear to provide sufficient theoretical foundations to explain all of the trends in our findings. In addition to basic conceptions of how children may model the behavior of their elders, our understanding might be enhanced by considering the alternative theoretical foundations described earlier. In accordance with attachment and self-determination theories, for example, aversive parenting may weaken the child's ability to assemble proper expectations of functional relationships, either at home or in the peer group. In this sense, all psychological control dimensions, except the invalidation of feelings, may promote feelings of insecurity in the parent-child relationship (at least in Russia). Relationship insecurity breeds lower confidence in peer relationships, and aggression emerges as a prominent, preemptive line of defense against any perceived threats (Soenens et al., 2008). Both physical and relational aggression may be considered appropriate strategies for self-defense.

Psychological control may also engender significant negative emotion in children as they react to the very real manipulation of such strategies. Feelings of parental rejection may naturally flow from the divisive interactions which normally result. Anger and frustration are two prominent emotions which may result, which are consequently translated into higher levels of disruptive behavior with peers. Therefore, some dimensions of psychological control may be more easily interpreted by children as signs of parental rejection, which leads, by extension, to externalizing behaviors. Such a possibility is forwarded by Rohner's parental acceptance-rejection theory (PARTheory; Khaleque \& Rohner, 2002), which posits that psychological adjustment of children is directly correlated with the degree of acceptance or rejection they feel from their parents. Aggression is one of the important indices of psychological maladjustment that children exhibit when denied parental acceptance. Importantly, this theory is proposed to hold for children in any part of the world. Accordingly, psychological control may engender feelings of parental rejection in Russian preschoolers, which leads to externalizing behavior in the form of both physical and relational aggression. Even if some children naturally begin life with more aggressive behavioral problems, a negative relationship with parents would exacerbate these issues. Russian preschool boys would appear to be more vulnerable in this regard.

Similarly, children's social information processing may be dramatically affected by the ongoing onslaught of their parents' psychological control strategies. Parents who practice psychological control may subtly communicate distrust in the child's ability to function autonomously and make proper choices. Misbehavior of any sort may be perceived by parents to be malicious in intent, and children may come to expect that all potential provocations are intentional and should be dealt with harshly. Some research suggests that this may vary by gender of child. In particular, the tendency of boys to properly process social information may be more adversely affected by negative parenting than that of girls, who may turn to other relationships, such as friendships, as their primary source of learning social information-processing skills (Nelson \& Coyne, 2009). With all of these possibilities, further research is clearly needed to explore the nature of the mechanisms by which parenting may be translated into relational aggression. We may expect that no one mechanism may predominate.

Of course, in considering these parent-child associations, we cannot establish the direction of effect between parent and child. Most likely, it is bidirectional (Deater-Deckard \& Dodge, 1997). It may be that parents respond to aggressive children with more aversive parenting, such as psychological control. In turn, preschool children, who are recipients of limited social interaction with peers, will likely reflect some of the lessons learned in parent-child relationships. Longitudinal work would be useful to better illuminate associations between parental psychological control and child behavior over time.

The results of this study are suggestive, but limited in several ways. First, our sample was limited to preschool children, and the connection between psychological control and aggression may be different in older age groups. In fact, most studies of psychological control and relational aggression have focused on early- and middle-childhood samples. As noted above, Barber $(1996,2002)$ has emphasized the detrimental effects of psychological control for adolescents in particular. Second, we involved a group of parents who were relatively well educated, and it is not clear if the findings will apply for lower-SES parents within Russia or other cultures. Third, parental self-ratings have potential disadvantages. In particular, social desirability may lead parents to overreport positive parenting behaviors while underreporting aversive parenting practices. This approach might be best supplemented by the child's ratings of the parent's behavior (Barber, 1996), or perhaps the addition of spouse reports, which have demonstrated utility in other reports with cultural samples (Yang et al., 2004). Such an approach would assess multi-method agreement and allow a better sense of the relative value of alternative informants.

Another consideration is that the relatively small sample does not allow us much room to answer a number of important questions about these dimensions. For example, it would be useful to gauge whether there may be different behavioral profiles in regard to psychological control in parents. Some parents may engage in high levels of all dimensions of psychological control, whereas other parents may engage in uniformly low levels of all dimensions. Also likely, however, is that a number of parents engage in jagged profiles, where they favor certain forms of psychological control and neglect others. These varied profiles may uniquely interact with children's temperaments to yield a range of child outcomes, or different levels of maladjustment. A natural statistical paradigm to explore this question is cluster analysis.

Given how highly correlated some dimensions of psychological control tend to be, it may be argued that it is difficult and perhaps not meaningful to separate these dimensions in future research. It is notable that four of five dimensions had significant associations with childhood aggression. Accordingly, the message may be that psychological control dimensions tend to be uniformly negative in their influence, as Barber 
(1996) has suggested. However, just as some forms of authoritarian parenting may be more detrimental than others (e.g., corporal punishment as compared to strictness), future research may yet reveal that some forms of psychological control are especially pertinent in child development.

In recent years, there has been a lot of controversy regarding spanking in the authoritarian parenting literature. Baumrind, Larzelere, and Owens (2010) have suggested that the effects of spanking are likely overstated, and we should not be too concerned about "normative spanking," in which parents engage in infrequent bouts of nonabusive spanking. The same question may be applied to psychological control. In other words, there may be dimensional effects, dose-dependent effects (regardless of dimensions tapped), or a combination of the two. Longitudinal studies, like that employed by Baumrind and colleagues, will be the most useful in identifying whether psychological control, as a unified or multidimensional construct, is associated with long-term child maladjustment. Practically speaking, however, it makes sense to promote positive discipline practices in parenting over spanking or psychologically controlling methods. Reduction of potential risk and promotion of established protective factors in parenting will increase the chance of developmental success in children.

In summary, this study provides additional evidence that parental psychological control is clearly a construct worth further study, particularly in the study of childhood aggression. Consistent with previous research in the U.S. and China, parental psychological control is associated with childhood aggression in Russia. Accordingly, psychological control may be a universally negative form of parenting across cultures. Further study is needed to more clearly assess how this form of parenting is interpreted by children and incorporated into their feelings and behaviors.

\section{References}

Archer, J., \& Coyne, S. M. (2005). An integrated review of indirect, relational, and social aggression. Personality and Social Psychology Review, 9, 212-230.

Bandura, A. (1971). Social learning theory. New York, NY: General Learning Press.

Barber, B. K. (1996). Parental psychological control: Revisiting a neglected construct. Child Development, 67, 3296-3319.

Barber, B. K. (2002). Intrusive parenting: How psychological control affects children and adolescents. Washington, D.C.: American Psychological Association.

Baumrind, D., Larzelere, R. E., \& Owens, E. B. (2010). Effects of preschool parents' power assertive patterns and practices on adolescent development. Parenting: Science and Practice, 10, 157-201.

Bayer, J. K., Sanson, A. V., \& Hemphill, S. A. (2006). Parent influences on early childhood internalizing difficulties. Journal of Applied Developmental Psychology, 27, 542-559.

Bowlby, J. (1988). A secure base: Parent-child attachment and healthy human development. New York, NY: Basic Books.

Bronfenbrenner, U. (1970). Two worlds of childhood: U.S. and U.S.S.R. New York, NY: Russell Sage Foundation.

Cairns, R. B., Cairns, B. D., Neckerman, H. J., Ferguson, L. L., \& Gariepy, J. L. (1989). Growth and aggression: 1. Childhood to early adolescence. Developmental Psychology, 25, 320-330.

Casas, J. F., Weigel, S. M., Crick, N. R., Ostrov, J. M., Woods, K. E., Jansen Yeh, E. A., et al. (2006). Early parenting and children's relational and physical aggression in the preschool and home contexts. Applied Developmental Psychology, 27, 209-227.

Chang, L., Schwartz, D., Dodge, K. A., \& McBride-Chang, C. (2003). Harsh parenting in relation to child emotion regulation and aggression. Journal of Family Psychology, 17, 598-606.

Crick, N. R., Casas, J. J., \& Mosher, M. (1997). Relational and overt aggression in preschool. Developmental Psychology, 33, 579-588.

Crick, N. R., \& Dodge, K. A. (1994). A review and reformulation of social informationprocessing mechanisms in children's social adjustment. Psychological Bulletin, 115, 74-101.

Crick, N. R., \& Grotpeter, J. K. (1995). Relational aggression, gender, and social-psychological adjustment. Child Development, 66, 710-722.

Davis, B. T., Hops, H., Alpert, A., \& Sheeber, L. (1998). Child responses to parental conflict and their effect on adjustment: A study of triadic relations. Journal of Family Psychology, 12, 163-177.
Deater-Deckard, K., \& Dodge, K. A. (1997). Externalizing behavior problems and discipline revisited: Nonlinear effects and variation by culture, context, and gender. Psychological Inquiry, 8, 161-175.

Deci, E. I., \& Ryan, R. M. (2000). The 'what' and 'why' of goal pursuits: Human needs and the self-determination of behavior. Psychological Inquiry, 11, 227-268.

Feshbach, N. D. (1969). Sex differences in children's modes of aggressive responses toward outsiders. Merrill-Palmer Quarterly, 15, 249-258.

Galen, B. R., \& Underwood, M. K. (1997). A developmental investigation of social aggression among children. Developmental Psychology, 33, 589-600.

Hart, C. H., Nelson, D. A., Robinson, C. C., Olsen, S. F., \& McNeilly-Choque, M. K. (1998), Overt and relational aggression in Russian nursery-school-age children: Parenting style and marital linkages. Developmental Psychology, 34, 687-697.

Hart, C. H., \& Robinson, C. C. (1995). Parental psychological control: An instrument for early childhood. Unpublished manuscript.

Horn, J. L., \& McArdle, J. J. (1992). A practical and theoretical guide to measurement invariance in aging research. Experimental Aging Research, 18, 117-144.

Khaleque, A., \& Rohner, R. P. (2002). Perceived parental acceptance-rejection and psychological adjustment: A meta-analysis of cross-cultural and intracultural studies. Journal of Marriage and Family, 64, 54-64.

Komaroff, E. (1997). Effects of simultaneous violations of essential Tau equivalence and uncorrelated error on coefficient alpha. Applied Psychological Measurement, 21 337-348.

Kuppens, S., Grietens, H., Onghena, P., \& Michiels, D. (2009). Associations between parental control and children's overt and relational aggression. British Journal of Developmental Psychology, 27, 607-623.

Lagerspetz, K. M. J., Björkqvist, K., \& Peltonen, T. (1988). Indirect aggression in girls and boys, In L. R. Huesmann (Ed.), Aggressive behavior: Current perspectives (pp. 131-150). New York, NY: Plenum.

Loukas, A., Paulos, S. K., \& Robinson, S. (2005). Early adolescent social and overt aggression: Examining the roles of social anxiety and maternal psychological control. Journal of Youth and Adolescence, 34, 335-345.

McNeilly-Choque, M. K., Hart, C. H., Robinson, C. C., Nelson, L. J., \& Olsen, S. F. (1996) Overt and relational aggression on the playground: Correspondence among different informants. Journal of Research in Childhood Education, 11, 47-67.

McShane, K. E., \& Hastings, P. D. (2009). The new friends vignettes: Measuring parental psychological control that confers risk for anxious adjustment in preschoolers. International Journal of Behavioral Development, 33, 481-495.

Mills, R. S. L., Freeman, W. S., Clara, I. P., Elgar, F. J., Walling, B. R., \& Mak, L. (2007) Parent proneness to shame and the use of psychological control. Journal of Child and Family Studies, 16, 359-374.

Muthén, L. K., \& Muthén, B. O. (2001). Mplus user's guide (2nd ed.). Los Angeles, CA: Muthén \& Muthén.

Nelson, D. A., \& Coyne, S. M. (2009). Children's intent attributions and feelings of distress: Associations with parenting practices of mothers and fathers. Journal of Abnormal Child Psychology, 37, 223-237.

Nelson, D. A., \& Crick, N. R. (2002). Parental psychological control: Implications for childhood physical and relational aggression. In B. K. Barber (Ed.), Intrusive parenting: How psychological control affects children and adolescents (pp.168-189). Washington, D.C.: American Psychological Association.

Nelson, D. A., Hart, C. H., Yang, C., Olsen, J. A., \& Jin, S. (2006). Aversive parenting in China: Associations with child physical and relational aggression. Child Development, 77, 554-572.

Nelson, D. A., Mitchell, C., \& Yang, C. (2008). Intent attributions and aggression: A study of children and their parents. Journal of Abnormal Child Psychology, 36, 793-806.

Nelson, D. A., Robinson, C. C., \& Hart, C. H. (2005). Relational and physical aggression of preschool-age children: Peer status linkages across informants. Early Education and Development, 16, 115-139.

Rapee, R. M. (1997). Potential role of childrearing practices in the development of anxiety and depression. Clinical Psychology Review, 17, 47-67.

Schmitt, N. (1996). Uses and abuses of coefficient alpha. Psychological Assessment, 8, 350-353.

Soenens, B., Vansteenkiste, M., Goossens, L., Duriez, B., \& Niemiec, C. (2008). The intervening role of relational aggression between psychological control and friendship quality. Social Development, 17, 661-681.

Stevens, J. (1996). Applied multivariate statistics for the social sciences. Mahwah, NJ: Lawrence-Erlbaum.

Weiss, B., Dodge, K. A., Bates, J. E., \& Pettit, G. S. (1992). Some consequences of early harsh discipline: Child aggression and a maladaptive social information processing style. Child Development, 63, 1321-1335.

Widaman, K. F., \& Reise, S. P. (1997). Exploring the measurement invariance of psychological instruments: Applications in the substance use domain. In K. J Bryant, M. Windle, \& S. G. West (Eds.), The science of prevention: Methodological advances from alcohol and substance abuse research (pp. 281-324). Washington, D.C.: APA.

Yang, C., Hart, C. H., Nelson, D. A., Porter, C. L., Olsen, S. F., Robinson, C. C., et al. (2004) Fathering in a Beijing, Chinese sample: Associations with boys' and girls' negative emotionality and aggression. In R. D. Day, \& M. E. Lamb (Eds.), Conceptualizing and measuring father involvement (pp. 185-215). Mahwah, NJ: Lawrence Erlbaum. 